\title{
Research on Ultrasonic Phased Array Imaging Based on Information Fusion and GPU Technologies
}

\author{
Zhan Xianglin, Cai Yujie, Liu Dai \\ College of Aeronautical Automation \\ Civil Aviation University of China \\ Tianjin, China \\ xlzhan@cauc.edu.cn
}

\begin{abstract}
It is a big challenge to get complete accurate information as well as deal with huge amount of signal information when an ultrasonic phased array (UPA) instrument is used to test aircraft components. To solve this problem, a method that combined information fusion technology with Graphic Processing Unit (GPU) is presented in this paper. At first, the information fusion algorithm is introduced. Then, a hardware platform based on GPU is established and is used to carry out various calculations of the algorithm because GPU has a lot of parallel processes. Finally, UPA signals are simulated, which are processed by the proposed information fusion algorithm on the GPU platform. The experiment results suggest that information fusion algorithm can get more complete information characteristics from the echo signals wheareas GPU can improve data processing speed. Therefore, the information fusion technology combined with GPU can improve utilization of UPA signals and give more accurate and reliable description to the reality testing environment, which provide a solution to the challenge mentioned above.
\end{abstract}

Keywords- ultrasonic phased array ; nondestructive testing Graphic Processing Unit; Information Fusion; signal processing

\section{INTRODUCTION}

Before an aircraft is put into use, the performance of its components must meet airworthiness standards. Furthermore, cracks may appear in the structure or on the surface of the component after the airplane flies a certain miles due to the natural environment or improper maintenance. These injuries can affect flight performance or even flight safety of the aircraft .So the aircraft must be inspected or maintained regularly. But it is very difficult to conduct a comprehensive inspection because the aircraft has a large number of composite components with complex structures. However, the use of ultrasonic phased array (UPA) technology can inspect a composite component without moving the probe. Through controlling time delay of transmission and reception of each element, the inspection ultrasound beam can deflect and focus at the inspected area. Because multiple angles and focus depths can be changed electronically, testing efficiency and accuracy are improved. Correspondingly, the workload of testing personnel is reduced.

UPA technology was proposed in the eighties last century. It was mainly used in medical ultrasound imaging diagnosis [1]. Since the electronic technology develops very quickly, the UPA technology has been widely used in the industrial filed too. Normally, a UPA system mainly consists of two parts, an ultrasonic array transducer and an electronic control system [2]. The UPA transducer is composed of a set of independent array elements. According to a certain timing rule that is controlled by the electronic system, selected array elements emit ultrasonic waves. The waves superpose to form a new wave front based on the acoustic interference principle. Similarly, during reception of reflected waves, the selected elements are controlled to receive the echoes according to the same timing rule that is used in transmission. All the echoes are synthesized and are displayed in the appropriate wave form in the instrument screen. In fact, the UPA technology can achieve dynamic focusing and scanning in high speed by the means of electronically controlling phase delay among transducer array elements.

In this paper, a method that combined information fusion technology with Graphic Processing Unit (GPU) is presented. First, the principle of information fusion algorithm is described. Then, GPU is introduced and a hardware platform based on GPU is established. It is used to carry out various calculations of the algorithm. And then, experiments are carried out. UPA signals are simulated and are processed by the proposed information fusion algorithm on the GPU platform. Finally, some conclusions are drawn and future researches are discussed.

\section{THE PRINCIPLE OF INFORMATION FUSION ALGORITHM}

It is observed that echo information obtained by individual UPA element has a little bit different signal characteristics because each element is independent. This indicates that inspection information cannot be completely described by the samples. In addition, amplitude attenuation of the ultrasonic in the composite is very large. Therefore the ultrasound echo amplitude obtained by the UPA system is very small, which is not easy to process. Furthermore, the information data obtained by the UPA system is very too large. How to effectively process the data is a big challenge. To solve the technology difficulties mentioned above, information infusion algorithm is introduced. 


\section{A. Information Fusion principle}

The basic principle of information fusion is to simulate the function of an integrated human brain that can process information by taking full advantage of multi-source information. In fact, to obtain consistency explain or describe of the measured object, the brain can control and use multiple sources of information, combining the redundancy and complementarity in space or time based on a certain criteria [3].

There is no unified structure and classification form in information fusion research field. According to the abstraction level of information processing hierarchy in the multi-sensor system, information fusion can be classified as pixel-level, feature-level and decision-level fusion [4]. Pixel-level fusion is the lowest level of integration. The raw data obtained by the sensor are not processed or with very little processing. Then, they are just directly put in fusion. Since the data basically are not treated before fusion, more of the original details are preserved than other levels. But fusion results have the highest precision, giving people a more accurate and comprehensive understanding. On the other hand, the data processing capacity of this fusion method has poor real-time performance and requires a lot of communication bandwidth. Feature-level fusion is to extract features from the raw data collected by each sensor at first. And then these features are fused. This kind of fusion is able to increase the accuracy of features and is helpful for real-time processing because information has been processed before fusion. But its disadvantage is the fusion precision is lower than that of the pixel-level fusion. Decision-level fusion is the highest level of fusion. The final decision is resulted from local decision that comes from multi-source information directly. This kind of algorithm has good fault tolerance and real-time performance, and can be used to process information from heterogeneous sensors. More importantly, when one or more sensors fail, the algorithm can still work properly. But its disadvantage is the high cost of pre-treatment, and a relatively small bandwidth is required. From the above analysis, it can be seen that feature-level fusion is an intermediate level between the pixel-level and decision-level approach.

\section{B. An information fusion algorithm Based on Bayesian Estimation}

To realize information fusion process, one or several kinds of arithmetic algorithms are needed. Information fusion algorithms can be divided into the following four categories specifically: estimation methods, classification methods, reasoning method and artificial intelligence methods [5]. Generally, estimation methods include weighted average method, least squares method, and Kalman filtering method. Classification methods consist of parametric template method and cluster analysis. And commonly used reasoning methods are Bayesian inference and evidence theory. Artificial intelligence methods comprise expert systems, neural networks, fuzzy logic, and genetic algorithms. They have advantages in case of processing a lot of information fusion or nonlinear and uncertain problems. In this paper, an information fusion method based on Bayesian estimation is introduced.

First, an echo received from an array element is made up of $n$ points, and the value of the points is the amplitude displayed on the instrument screen. Also, the echo can be seen as a function, and its probability density curve $p(x)$ can be regarded as its eigenfunction. Suppose there are $m$ echoes. All the echoes comprise a matrix, whose column is one echo.

Second, confidence distance measure $d_{i j}(i, j=1,2, \ldots, n)$ of $\mathrm{m}$ echoes are computed based on (1).

$$
d_{i j}=2 \int_{x_{i}}^{x_{j}} p_{i}\left(x \mid x_{i}\right) d x
$$

where $p_{i}\left(x \mid x_{i}\right)$ is the conditional probability density when $i^{\text {th }}$ sample value is $x_{i}$. It can be defined as (2).

$$
p_{\mathrm{i}}\left(x \mid x_{i}\right)=\exp \left\{-\left(\frac{x-x_{i}}{\sigma_{i}}\right)^{2} / 2\right\} / \sqrt{2 \pi \sigma_{i}}
$$

Where $\sigma_{i}$ is the variance of $i^{\text {th }}$ sample.

Third, a relationship matrix can be established as (3).

$$
R=\left(\begin{array}{ccc}
r_{11} & \ldots & r_{1 m} \\
\vdots & \cdots & \vdots \\
r_{n 1} & \ldots & r_{n m}
\end{array}\right)
$$

where $r_{i j}$ is the correlation coefficient of $i^{\text {th }}$ sample and $j^{\text {th }}$ sample, and can be defined as (4).

$$
r_{\mathrm{i} j}=\left\{\begin{array}{ll}
1_{j} & d_{\mathrm{i} j} \leq \beta_{\mathrm{i} j} \\
0 ; & d_{i \tilde{j}} \geq \beta_{\mathrm{i} j}
\end{array} .\right.
$$

where $\beta_{i j}$ is the limit value of $d_{i j}$.

Four, suppose $d\left(x_{1}, x_{2}, \ldots, x_{n}\right)$ is the decision function, where $x_{1}, x_{2}, \ldots, x_{n}$ are the points composed of one echo. The desired fusion result is expressed as $\mu$, but we cannot know it. What we can do is to approximate it as much as possible. The relationship between $\mu$ and $d$ can be defined as the risk function defined as (5).

$$
R\left(\mu \mid d_{i}\right)=E_{\mu}\left\{L\left(\mu, d\left(x_{1}, x_{2}, \ldots, x_{n}\right)\right)\right\} .
$$

where $L(\mu, d)$ is the quadratic form of loss function and can be defined as (6).

$$
L(\mu, d)=\left[\mu-d\left(x_{1}, x_{2, \ldots s} x_{n}\right)\right]^{2} .
$$

Then, the Bayesian estimation of $\mu$ is $\hat{\mu}$ :

$$
\hat{\mu}=\int_{\emptyset} \mu p\left(\mu \mid\left(x_{1}, x_{2}, \ldots, x_{n}\right)\right) d \mu .
$$

If $p\left(\mu \mid\left(x_{1}, x_{2, \ldots,} x_{n}\right)\right.$ obey the normal distribution $\mathrm{N}\left(\mu_{N}, \sigma_{N}^{2}\right), \mu_{N}$ is the mean value, $\sigma_{N}^{2}$ is the variance. Then, 


$$
p\left(\mu \mid\left(x_{1}, x_{2}, \ldots, x_{n}\right)\right)=\frac{1}{\sqrt{2 \pi} \sigma_{N}} \exp \left\{-\frac{1}{2}\left(\frac{\mu_{N}-\mu_{0}}{\sigma_{0}}\right)^{2}\right\} .
$$

where $\sigma_{0}$ is the standard deviation of all samples whereas $\mu_{0}$ is the mean value of all samples.

Therefore,

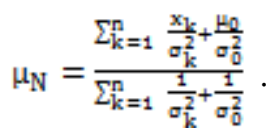

Based on (7), it can be deferred that $\mu_{N}$ is also the Bayesian estimation of $\mu$. Therefore, $\mu_{N}$ is the optimum fusion results of the echoes that collected by UPA elements.

\section{THE HARDWARE PLATFORM BASED ON GPU}

The full name of GPU is Graphic Processing Unit. In fact, it is the graphic processor. GPU is a dedicated integrated circuit chip used for parallel computation [6]. Since the invention of the computer, it has been committed to speed up computer processing speed. Comparing with those in 10 years before, multi-core CPU and multi-threading technology have been appearing. Accordingly, computing speed of the computer has also been greatly improved. However, due to the limits of physical devices, such as the bottleneck of a single processor speed, CPU is still a serial device essentially. In many applications, fast computing speed is also needed. Though many scholars have proposed a lot of simplified calculation methods, it still takes an existing computer a few hours, a few days or even weeks to complete the calculation. However, these large number and complex calculations can be done quickly by GPU parallel computing. Compared to the CPU, GPU owns faster computational speed and larger memory bandwidth [7].

From another point of view, GPU is the computing core of a video card. It is a device designed for graphics techniques. And it does not open directly to the programmer, but is a closed model, i.e., computer tasks are mapped to textures rendering by packaging data into pairs.

In this paper, a hardware platform based on GPU technology has been established. The block diagram is displayed in Fig .1.

\section{EXPERIMENTS}

\section{A. Introduction to Experimental conditions}

Experimental simulation hardware platform is an IPC, a Graphics card (Intel Ivy Bridge Graphics Controller (1 GB / Lenovo)) and GPU (DDR5). Software environment is MATLAB programming. The number of UPA elements is 128. Parameters of an array element are as follows: width is $0.2 \mathrm{~mm}$, height is $5 \mathrm{~mm}$. The focus of the array is $(0,0$, 30) $\mathrm{mm}$. Experimental object is a stainless steel, whose length is $20 \mathrm{~cm}$, width is $10 \mathrm{~cm}$, and thickness is $0.5 \mathrm{~cm}$.

\section{B. Experimental procedures}

- $\quad$ Selection. Ultrasound field is simulated by Field2 programming, setting experiment parameters of the transmission elements from NO. 1 to NO.4. Then, four wave forms, each of which is composed by 1088 dots, are shown in Fig .2.

- Information fusion algorithm is adopted to process the above signals;

- $\quad$ CPU and GPU are used respectively to simulate information fusion algorithm by MATLAB software.

- Final simulation results are got and shown in Fig .3 and Fig .4.

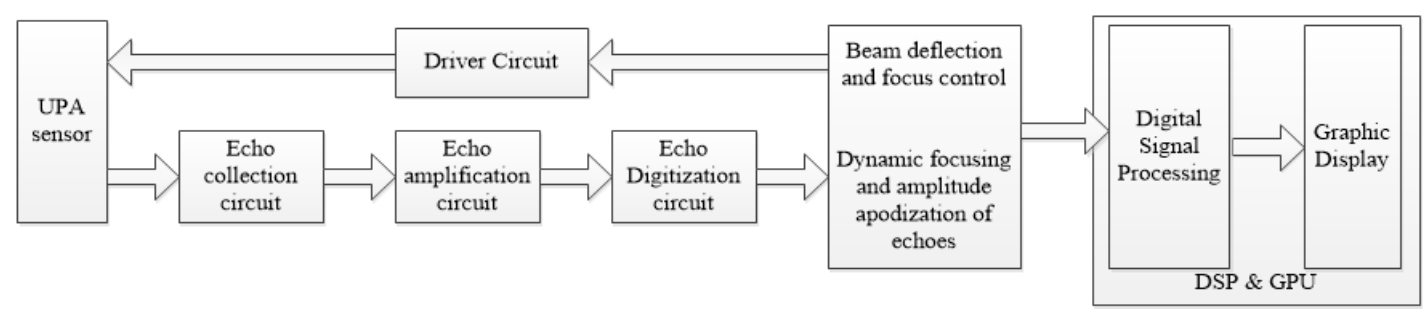

Figure 1. Block diagram of the hardware platform based on GPU.

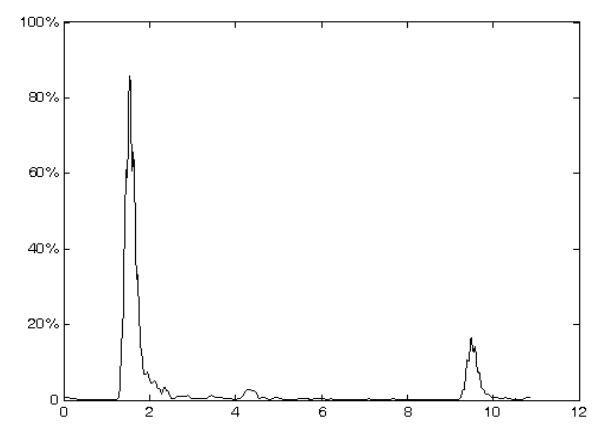

(a) Emission images of $1 \#$ element.

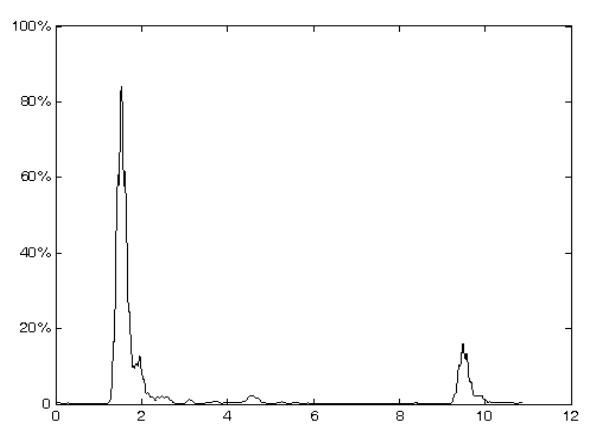

(b) Emission images of 2\# element. 


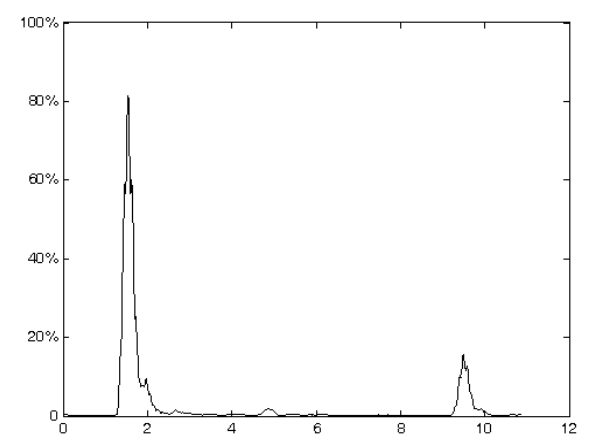

(c) Emission images of 3\# element.

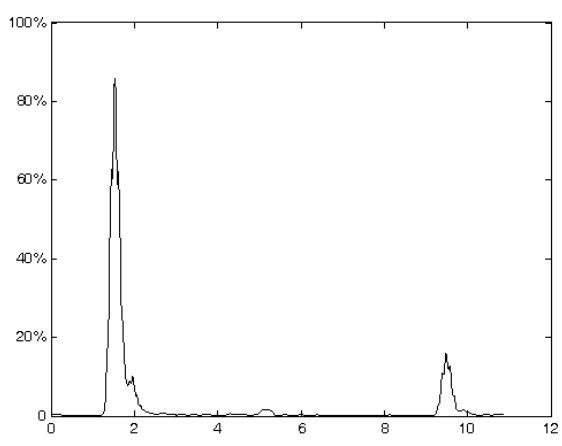

(d) Emission images of $4 \#$ element.

Figure 2. Emission Images of $1 \#$ to $4 \#$ array elements.

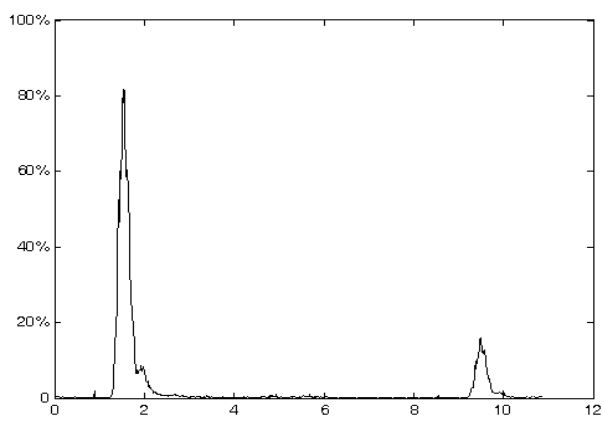

Figure 3. Graph obtained on CPU

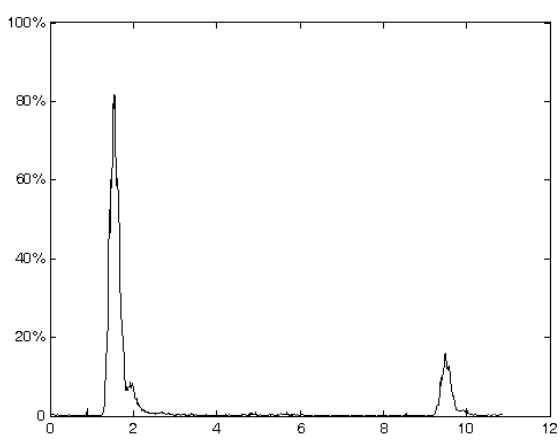

Figure 4. Graph obtained on GPU.

From Fig .2, we can see four graphics are not the same, that is to say, signal characteristics of each array element collected have difference. In order to utilize useful information characteristics comprehensively and to exclude error message features, information fusion algorithm is use.More accurate and reliable description about reality environment is improved.

Fig .3 and Fig .4 are the image results using information fusion technology by MATLAB programming on the CPU and GPU respectively. We can see the effect of the two images is same. However, adopting information fusion algorithm to process 1088 points for each graphic of the four signals is a large amount of computation. The simulation on CPU needs 18 minutes. But the same simulation on GPU only needs 2 minutes.Therefore, simulation on GPU can reduce the computation time greatly. Therefore, the expected results of the simulation experiments are achieved.

\section{CONCLUSION}

It is very difficult for a UPA nondestructive testing of an aircraft comp to get complete but accurate information from a great deal of signal information.

In this paper, a method that combined information fusion technology with Graphic Processing Unit (GPU) is proposed. The experiment results indicate that this new method can improve the utilization of signal and get more accurate and reliable description to the reality environment.

\section{ACKNOWLEDGMENT}

The authors would like to thank National Natural Science Foundation of China (Grant NO. 61102097 and 61405246), Tianjin Science and Technology Support Program, China (Grant NO. 11ZCGHHZ00700) and Central Universities Fundamental Research Grant (SY-1418 and 3122014D023) to support.

\section{REFERENCES}

[1] Zhou Qi, Liu Fangjun, and Li Zhijun, "Ultrasonic phased array imaging technology and applications" Ordnance Material Science Engineering, vol. 25, 2002,pp.34-37.

[2] Chen Yanhong, "Dynamic focusing ultrasonic phased array technology research," Chengdu: Southwest Jiaotong University, 2014.

[3] Han Zengqi, Yu Junjie, and Li Ningxia, "The review of information fusion technology,". Journal of Intelligence, vol. 29(S1), 2010, pp.110-114.

[4] He You, Wang Guohong, and $\mathrm{Lu}$ da, "Use of multi-sensor information fusion," Beijing: Electronic Industry Press, 2000.

[5] Tian Xiang, Tang Zhenmin, "Review of information fusion in urban rail transit system," Microcomputer Information, vol.21, 2005, pp.56-58.

[6] Wang Gang, Tang Jie, and Wu Gangshan, "Programming framework based on multi-GPU cluster," Computer Technology and Development, vol. 24, 2014, pp. 9-13.

[7] Li Xiaomin, Hou Chaohuan, snf Yan Shefeng, "Implementation formed GPU and CPU-based real-time collaboration beam," Application Research of Computers,vol. 28, 2011, pp. 1333-1335

[8] Kam M, Zhu X, and Kalata P, "Sensor fusion for mobile robot navigation," Proceedings of the IEEE Special Issue on Sensor Fusion, vol. 85, 1997, pp. 108-119.

[9] Wang Liang, Lv Weimin, Teng Kenan, and Li Jiazhe, "Research on equipment failure prediction based on data-driven technology," Computer Measurement \& Control, vol. 21, 2013 , pp. 2087-2105

[10] Pan Quan, Yu Xin, Cheng Yongmei, and Zhang Hongcai "Methods and advances in information fusion theory," Acta Automatica Sinica, vol. 29, 2003, pp.599-615. 